\title{
BMJ Open Assessing the impact of colonoscopy complications on use of colonoscopy among primary care physicians and other connected physicians: an observational study of older Americans
}

\author{
Nancy L Keating, ${ }^{1,2}$ A James O’Malley, ${ }^{3}$ Jukka-Pekka Onnela, ${ }^{4}$ Bruce E Landon ${ }^{1,6}$
}

To cite: Keating NL, James O'Malley A, Onnela J-P, et al. Assessing the impact of colonoscopy complications on use of colonoscopy among primary care physicians and other connected physicians: an observational study of older Americans. BMJ Open 2017;7:e014239. doi:10.1136/ bmjopen-2016-014239

- Prepublication history and additional material are available. To view these files please visit the journal online (http://dx.doi org/10.1136/bmjopen-2016014239).

Received 12 September 2016 Revised 26 January 2017 Accepted 20 March 2017

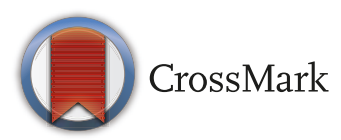

For numbered affiliations see end of article.

Correspondence to

Dr Nancy L Keating;

keating@hcp.med.harvard.edu

\section{ABSTRACT}

Objectives Psychological biases can distort treatment decision-making. The availability heuristic is one such bias, wherein events that are recent, vivid or easily imagined are readily 'available' to memory and are therefore judged more likely to occur than expected based on epidemiological data. We assessed if the occurrence of a serious colonoscopy complication for a primary care physician's patient influenced colonoscopy rates for the physician's other patients.

Design Longitudinal study with time-varying exposure variables.

Setting/participants Individuals living in 51 hospital referral regions across the USA identified based on enrolment in fee-for-service Medicare during 2005-2010. We assigned patients to a primary care physician based on office visits during the prior 2 years.

Exposures For each physician in each month, we calculated the proportion of patients assigned to them who had a colonoscopy. We identified two serious complications of which the primary care provider would very likely be aware: gastrointestinal bleed or perforation leading to hospitalisation or death within 14 days of colonoscopy.

Main outcome measures We employed Poisson regression models including physician fixed effects to assess the change in number of colonoscopies in the four quarters following an adverse colonoscopy event. Results We identified 5360191 patients assigned to 30704 physicians. 4864 physicians (16\%) had at least one patient with an adverse event. The estimated change in the quarterly number of colonoscopies among physicians' patients was significantly lower in quarter 2 following an adverse colonoscopy event (change $=-2.1 \%$ $(95 \% \mathrm{Cl}-3.4$ to $-0.8 \%))$, before returning to the rate expected in the absence of an adverse event.

Conclusions Having a patient experience a serious adverse colonoscopy event was associated with a small and temporary decline in colonoscopy rates among a physician's other patients. This finding provides empirical evidence for the influence of notable adverse events on care, possibly due to the availability heuristic.
Strengths and limitations of this study

- We studied a large representative cohort of patients and their physicians allowing us to assess the consequences of infrequent but very serious adverse events of colonoscopy, a frequently recommended screening procedure.

- We used a longitudinal study design with timevarying exposure variables to adjust for patient and physician factors in our longitudinal data.

- Limitations included our focus on Americans aged 65 years and older, the possibility of misattribution of patients to physicians and the lack of direct information about physicians' and patients' decision-making processes.

\section{INTRODUCTION}

A physician's recommendation is an important determinant of whether a patient undergoes recommended screening and preventive testing such as colorectal cancer screening. ${ }^{1-3}$ Traditionally, cancer screening is a core component of primary care, and adherence to screening recommendations is a key quality indicator for primary care physicians and practices. Colorectal cancer screening is particularly effective, and expanded screening is credited as contributing to at least some of the declining incidence and mortality from this condition. ${ }^{45}$ The United States Preventative Services Task Force recommends screening for colorectal cancer for adults beginning at age 50 for average-risk individuals. ${ }^{6}$ Although there are several accepted methods for colorectal cancer screening, including annual faecal occult blood screening and flexible sigmoidoscopy every 5 years, most primary care physicians and gastroenterologists favour colonoscopy as the preferred method of screening. ${ }^{7}$ 
Colonoscopy, however, has potential risks, which have been increasingly highlighted by guideline panels. These risks include complications from the procedure, ${ }^{8}$ as well as the possibility of false positive tests and overdiagnosis and overtreatment.

Evidence suggests that psychological biases can distort treatment decision-making relative to a traditional utility maximisation reference. ${ }^{9}$ The availability heuristic is one such bias wherein events that are recent, vivid or easy to imagine are readily 'available' to memory and are therefore judged to be more likely to occur than would be expected based on epidemiological data. ${ }^{10}$ Yet, few data are available that quantify the impact of such biases on physician decision-making and the care that is delivered to patients. Two prior studies have provided some evidence for the effect of availability bias on diagnostic judgements, but these studies were small and limited to resident physicians. ${ }^{11} 12$ Expanding the evidence base regarding the rational and non-rational forces driving physician behaviour will help to identify specific types of clinical decisions that might be influenced by such biases, which could allow physicians and organisations to design interventions to overcome biases when they are likely to occur. In addition, such data could help to identify opportunities for improving physicians' understanding of clinical evidence and how that evidence is shared with patients making decisions about care.

We used administrative data from the US Medicare programme for beneficiaries and their physicians from 2005 through 2010 to assess for empirical evidence of cognitive bias in colorectal cancer screening. Specifically, we examined if the occurrence of a serious complication of screening colonoscopy among a primary care physician's patients influenced colonoscopy rates among the physician's other patients in subsequent months. We hypothesised that screening rates for physicians' patients would decrease in the period following experience with a patient having a serious adverse event. We next assessed if any effects of serious adverse events of screening on future screening differed for patients in a physician's panel who were older (vs younger) since the relative ratio of benefit to harm of colonoscopy screening is lower for patients older than 75 years and current guidelines recommend against routine screening for this group. ${ }^{6}$ We hypothesised that effects would be greater for a physician's older patients. We also assessed if the effects differed by physicians' experience in practice, hypothesising that younger (less experienced) physicians may be more impacted than others by a serious adverse event. Finally, we assessed if the serious adverse event changed screening behaviours for other primary care physicians within that primary care physician's practice as might be expected if physicians shared experiences of adverse events with their colleagues. We hypothesised that if overall effects were large, there may be similar but smaller effects among peers.

\section{METHODS}

\section{Data and subjects}

We used 100\% Medicare data from the inpatient, outpatient and carrier files for this analysis. Medicare is the national health insurance programme for Americans aged 65 years and older. We studied care for patients living in 50 hospital referral regions in the USA during 2005-2010; the regions were randomly sampled with probability proportional to their size; we also included the Boston hospital referral region. We identified all patients aged $\geq 65$ years who were continuously enrolled in parts A and B of fee-for-service Medicare for at least 1 year (or until death if $<1$ year) during the study period.

Because our focus was on screening behaviours of physicians for patients they treated, we assigned all patients to a physician for each month during 2006 through the end of 2010 based on the plurality of office visits for primary care services in the 2 years preceding that month. ${ }^{13}$ This strategy is similar to attribution algorithms used to assign patients to physicians in US Accountable Care Organizations. We assigned patients to physicians based on 2 years of data to provide more stable patient panels and to account for patients ageing into or leaving fee-for-service Medicare or dying. We weighted physician visits from the more recent year 0.67 and from the earlier year 0.33 . For example, for January 2007, the algorithm assigned patients to a physician using claims from February 2005 through January 2007, with visits during February 2005January 2006 weighted 0.33 and visits from February 2006 to January 2007 weighted 0.67 . The algorithm uses evaluation and management codes for face-to-face office visits and first assigns patients to generalist physicians (internal medicine, family practice, general practice, geriatrics); patients with no visits to generalists physicians were assigned to other medical specialists who might plausibly serve as their primary care physician. We assessed physician specialty based on the specialty code on the submitted claims, which may best reflect the type of care that they delivered to patients at that visit. ${ }^{14}$ We then focused analyses on primary care physicians (the generalist specialties described above) and medical specialists who may be providing primary care (cardiology, pulmonary, nephrology, infectious disease, endocrinology and rheumatology; we did not include gastroenterology to avoid including physicians who may also be performing a patient's colonoscopy). In preliminary analyses, findings were similar when including only primary care physicians.

Among 45652 physicians to whom patients were assigned from 2005 to 2010, we excluded 14323 physicians with fewer than 25 Medicare patients assigned to them in any month (based on the assignment algorithm over the 2-year window described above) and an additional 625 physicians were excluded by focusing on care during 2006-2010. The final cohort included 5360191 patients assigned to 30704 physicians.

We obtained information about physician age and sex from the American Medical Association Physician Masterfile. For each physician, we also identified peer physicians 
who were working in the same practice based on the tax identification number used for billing; we considered all other physicians billing under the same tax identification number as peers whose practice decisions might be influenced by their colleagues' experiences and behaviours. We assigned $1.0 \%$ of physicians who submitted claims under more than one tax identification number to the tax identification number for the first claim they submitted during the calendar year.

\section{Identifying colonoscopy and serious complications associated with colonoscopy}

We identified all patients who underwent screening or diagnostic colonoscopies in the outpatient setting (Medicare place of service codes 22, 24, 49) using procedure codes included in the online supplementary appendix table. ${ }^{8}$ If patients had more than one colonoscopy in a 1-year period, we only included the first occurrence. Prior work has examined complications of colonoscopy leading to emergency department visit or hospitalisation within 30 days of the procedure. ${ }^{8}$ But primary care providers may be unaware of relatively minor complications that may not come to their attention. Therefore, in this analysis, we focused on two serious complications that were highly likely to be associated with the colonoscopy and of which the primary care provider would very likely be aware: gastrointestinal bleed or perforation within 14 days of the colonoscopy that led to hospitalisation or death (online supplementary appendix table).

For each physician in each month from January 2006 through December 2010, we calculated the number of colonoscopies and the colonoscopy rate, defined as the number of colonoscopies that his/her assigned patients had in that month divided by all patients assigned to that physician in that month. We also identified each month during which a physician had a patient that experienced a serious complication.

\section{Patient involvement}

The research protocol was approved by the Harvard Medical School Committee on Human Subjects (\#23686). Patient consent was not obtained because our data, which were previously collected for billing purposes, did not include patient identifiers. Patients were not involved in the study design, although we studied a common procedure that most older Americans have been asked to consider for colorectal cancer screening. ${ }^{7}$

\section{Analyses}

We used a longitudinal study design with time-varying exposure variables to understand the impact of colonoscopy complications on future screening behaviours. This is akin to a difference-in-differences design in that we examined care with 5 years of longitudinal data, physicians served as their own control during months prior to any adverse event; physicians who had no adverse event in any month (84\% of physicians) also served as controls. We used fixed effects Poisson regression with a logarithmic link function to model the expected number of colonoscopies in each month during the study period. The models included fixed effects for each physician as well as indicator variables for each of the 60 study months (which adjusts for differences over time and/or seasonal differences in colonoscopy use) and four time-varying indicator variables reflecting the presence or absence of a colonoscopy adverse event in each of the four quarters before the month of interest. We also included the number of patients assigned to each physician in that month as the Poisson offset variable; this effectively serves as a denominator for the dependent variable (number of colonoscopies in a month), allowing us to interpret model coefficients as estimates of the change in the rate of colonoscopies among a physician's assigned patients. Because a patient will have at most one colonoscopy per quarter, the rate is essentially the proportion of all assigned patients who receive a colonoscopy per quarter.

In a second set of models, we conducted stratified analyses for patients aged $65-74$ and 75 years and older to assess if effects of an adverse colonoscopy event on future colonoscopies varied for younger versus older patients since for older patients the benefits of colonoscopies may be less and the risk of adverse events greater. We also ran a single model to test the statistical significance of the age group interaction.

In a third set of models, we stratified analyses by physician age (as a proxy for experience/years in practice) above or below the median to assess if effects of a serious adverse colonoscopy event were more pronounced for younger (less experienced) physicians. We also tested the statistical significance of the physician age interaction.

In a fourth model, we restricted to the 5513 practices with more than one physician in our cohort and included quarterly indicators reflecting an adverse event in one of the preceding four quarters for each physician as well as a second set of quarterly indicator variables reflecting presence or absence of a colonoscopy adverse event among other physicians practising in the same practice for each of the four prior quarters. This allowed us to assess whether an adverse colonoscopy event among a peer physician in the practice influenced a physician's colonoscopy ordering.

Finally, as a robustness check, we reran our models using the number of mammograms as the dependent variable as a falsification test since colonoscopy adverse events should not have any influence on breast cancer screening. To do this, we assessed if physicians whose patients had an adverse event related to colonoscopy had any temporal changes in their rate of screening mammography among women patients aged 65 years and older (we expected no changes). We identified screening mammography based on procedure codes (online supplementary appendix table).

In all models, the repeated (monthly) observations on physicians were accounted for by using generalised estimating equations using the identity as the working correlation matrix. The resulting SEs are robust to the 


\begin{tabular}{|c|c|}
\hline \multicolumn{2}{|l|}{ Characteristic } \\
\hline Physician age in years, mean (SD) & $50.5(11.0)$ \\
\hline \multicolumn{2}{|l|}{ Sex $(\%)$} \\
\hline Male & 73.3 \\
\hline Female & 26.7 \\
\hline \multicolumn{2}{|l|}{ Specialty (\%) } \\
\hline Primary care physician & 85.0 \\
\hline Medical specialist & 15.0 \\
\hline $\mathrm{N}$ assigned patients, mean (SD) & $122.5(121.9)$ \\
\hline Age of assigned patients in years, mean (SD) & $77.1(2.0)$ \\
\hline $\begin{array}{l}\text { Proportion of physicians' assigned patients } \\
\text { who are male, mean (SD) }\end{array}$ & $39.9(13.7)$ \\
\hline \multicolumn{2}{|l|}{$\begin{array}{l}\text { Race/ethnicity of physicians' assigned } \\
\text { patients }\end{array}$} \\
\hline Proportion who are white, mean (SD) & $83.9(23.6)$ \\
\hline Proportion who are black, mean (SD) & $8.9(18.0)$ \\
\hline Proportion who are Hispanic, mean (SD) & $3.1(9.5)$ \\
\hline $\begin{array}{l}\text { Hierarchical condition category score of } \\
\text { physicians' assigned patients, mean (SD) }\end{array}$ & $1.45(0.42)$ \\
\hline $\begin{array}{l}\text { Yearly number of colonoscopies among } \\
\text { physicians' assigned patients, mean (SD) }\end{array}$ & $10.2(16.6)$ \\
\hline \multicolumn{2}{|l|}{$\begin{array}{l}\text { Quarterly number of colonoscopies among } \\
\text { physicians' assigned patients, mean (SD) }\end{array}$} \\
\hline Among all patients & $2.5(3.4)$ \\
\hline Among patients aged $65-74$ years & $1.6(2.2)$ \\
\hline Among patients aged 75 years and older & $1.0(1.6)$ \\
\hline $\begin{array}{l}\text { Monthly number of colonoscopies among } \\
\text { physicians' assigned patients, mean (SD) }\end{array}$ & $0.8(1.4)$ \\
\hline
\end{tabular}

Patient and physician characteristics and characteristics of physicians' patients were calculated for each month that they were in the data set (physicians) or were attributed to a physician (patients) and averaged over all months that they were observed. Data on physician age and sex were missing for 334 physicians.

true relationship between the variance and the mean of the outcome variable, which are restrictively assumed to be equal when the outcomes have a Poisson distribution, and to the correlation structure among a physician's observations. Data on physician age and sex were missing for 334 physicians; however, because we included physician fixed effects in models to adjust for physician differences, we did not include these time-invariant variables, and therefore all physicians and patients are included in final analyses. The sponsor had no role in the research.

\section{RESULTS}

We identified 5360191 patients assigned to 30704 physicians practising in 21770 practices for which 5513 practices had more than one physician in our cohort. Characteristics of the patients and physicians are included in table 1. The mean age of the physicians was 50.5
$(\mathrm{SD}=11.0) ; 73.3 \%$ were male, and they had an average of 122.5 Medicare patients assigned $(\mathrm{SD}=121.9)$. Physicians were observed for a mean (SD) of 42.2 (12.4) months (range $=1-49$ ). Among assigned patients, approximately 10 patients had a colonoscopy in any year, consistent with the number expected for a test that is recommended once every 10 years. Overall, 6095 patients $(0.1 \%)$ experienced a serious adverse colonoscopy event between January 2006 and December 2010; 4864 physicians (16\%) had at least one patient with a serious adverse event; 951 $(3 \%)$ of physicians had two or more patients experience an adverse event.

In models with physician fixed effects, the estimated number of colonoscopies among physicians' patients following an adverse colonoscopy event was significantly lower by $2.1 \%$ (95\% CI -3.4 to -0.8 ) in quarter 2 following the adverse event (table 2 and figure 1), before returning to the number that would be expected in the absence of an adverse event. In stratified analyses comparing physicians' patients aged $65-74$ years versus aged 75 years and older, the association of an adverse colonoscopy event was generally similar to our primary model (table 2 ), and the interaction of quarter following an adverse colonoscopy event by patient age group was not statistically significant ( $p$ for interaction $=0.15$ ). In stratified analyses comparing younger versus older physicians, the association of an adverse colonoscopy event with fewer subsequent colonoscopies was observed only for younger physicians ( $p$ for interaction $=0.007$, table 2). When assessing for peer effects, there was no detectable decrease in the colonoscopy rates among other primary care physicians in the physicians' practices (all $\mathrm{p}>0.15$ ) (table 2).

In our falsification test assessing if the expected number of mammograms for a physician's patients changed in the quarters following an adverse colonoscopy event, we found no differences (table 3).

\section{DISCUSSION}

Having a patient experience a serious adverse event from colonoscopy was associated with a small and temporary decline in rates of colonoscopy among a physician's other Medicare patients. This finding provides empirical evidence for the influence of notable adverse events on care, possibly due to the availability heuristic. The negative impact is relatively modest for this clinical condition, wherein screening generally is supported by strong evidence; effects could be larger for other clinical conditions. The decline we observed was seen in the second quarter following the adverse event, which is consistent with the lag in obtaining colonoscopy from the time a physician recommends/orders it and it is completed, such that the lower likelihood of referring for screening might not be evident until several months after the adverse event. As more time from the adverse event passed, this effect disappeared, suggesting that more recent experience with no adverse events led physicians to return to their baseline rate of ordering. 
Table 2 Change in quarterly number of colonoscopies among physicians' patients following an adverse colonoscopy event among a physician's patient

\begin{tabular}{|c|c|c|}
\hline & $\%$ change $(95 \% \mathrm{Cl})^{*}$ & p Value* \\
\hline \multicolumn{3}{|l|}{ Primary model } \\
\hline Quarter 1 & $-0.7(-2.0$ to 0.7$)$ & 0.34 \\
\hline Quarter 2 & $-2.1(-3.4$ to -0.8$)$ & 0.002 \\
\hline Quarter 3 & $-0.9(-2.3$ to 0.4$)$ & 0.18 \\
\hline Quarter 4 & $0.0(-1.4$ to 1.4$)$ & 1.00 \\
\hline \multicolumn{3}{|c|}{ Model stratified by patient age (above/below 75 years)† } \\
\hline \multicolumn{3}{|c|}{ Patients 65-75years } \\
\hline Quarter 1 & $-0.1(-2.5$ to 2.3$)$ & 0.91 \\
\hline Quarter 2 & $-4.3(-6.6$ to -2.0$)$ & $<0.001$ \\
\hline Quarter 3 & $-1.1(-3.6$ to 1.4$)$ & 0.39 \\
\hline Quarter 4 & $-1.6(-4.0$ to 0.9$)$ & 0.21 \\
\hline \multicolumn{3}{|c|}{ Patients $>75$ years } \\
\hline Quarter 1 & $-3.4(-6.0$ to -0.7$)$ & 0.01 \\
\hline Quarter 2 & $-2.7(-5.3$ to -0.1$)$ & 0.04 \\
\hline Quarter 3 & $-3.5(-6.1$ to -0.7$)$ & 0.01 \\
\hline Quarter 4 & $-1.1(-3.8$ to 1.7$)$ & 0.43 \\
\hline \multicolumn{3}{|c|}{ Model stratified by physician experience (age above/below median) $\ddagger$} \\
\hline \multicolumn{3}{|c|}{ Physicians $<50.2$ years } \\
\hline Quarter 1 & $-1.2(-3.3$ to 0.9$)$ & 0.25 \\
\hline Quarter 2 & $-5.1(-7.3$ to -3.0$)$ & $<0.001$ \\
\hline Quarter 3 & $-2.4(-4.4$ to -0.4$)$ & 0.02 \\
\hline Quarter 4 & $-0.2(-2.4$ to 2.0$)$ & 0.85 \\
\hline \multicolumn{3}{|c|}{ Physicians $\geq 50.2$ years } \\
\hline Quarter 1 & $-0.6(-2.4$ to 1.1$)$ & 0.48 \\
\hline Quarter 2 & $-0.1(-1.9$ to 1.7$)$ & 0.93 \\
\hline Quarter 3 & 0.1 (-1.8 to 2.0$)$ & 0.93 \\
\hline Quarter 4 & $-0.1(-2.0$ to 1.8$)$ & 0.91 \\
\hline
\end{tabular}

Model including physicians' patients and patients of other physicians in their practice (among 5513 practices with two or more physicians)

\begin{tabular}{|lll}
\hline Physician & & \\
\hline Quarter 1 & $-0.8(-2.5$ to 0.8$)$ & 0.31 \\
\hline Quarter 2 & $-2.6(-4.1$ to -1.1$)$ & 0.001 \\
\hline Quarter 3 & $-1.0(-2.6$ to 0.7$)$ & 0.25 \\
\hline Quarter 4 & $0.8(-0.9$ to 2.4$)$ & 0.37 \\
\hline Physicians' practice peers & & \\
\hline Quarter 1 & $0.3(-0.4$ to 1.0$)$ & 0.39 \\
\hline Quarter 2 & $0.5(-0.2$ to 1.2$)$ & 0.16 \\
\hline Quarter 3 & $0.0(-0.7$ to 0.7$)$ & 0.95 \\
\hline Quarter 4 & $0.6(0.0$ to 1.3$)$ & 0.07 \\
\hline
\end{tabular}

Bolded values reflect statistical significance at two-sided $p<0.05$.

*Using fixed effects Poisson regression to model the number of colonoscopies. Models included fixed effects for each physician and indicators for study month as well as four indicator variables reflecting presence or absence of a colonoscopy adverse event in each of the four quarters before the month of interest. Models also include the number of patients assigned to the physician in that month, which serves as an offset variable allowing an interpretation of the dependent variable (number of colonoscopies) as a rate (number of colonoscopies per number of assigned patients).

tp for interaction $=0.15$.

$\neq \mathrm{p}$ for interaction $=0.007$. 


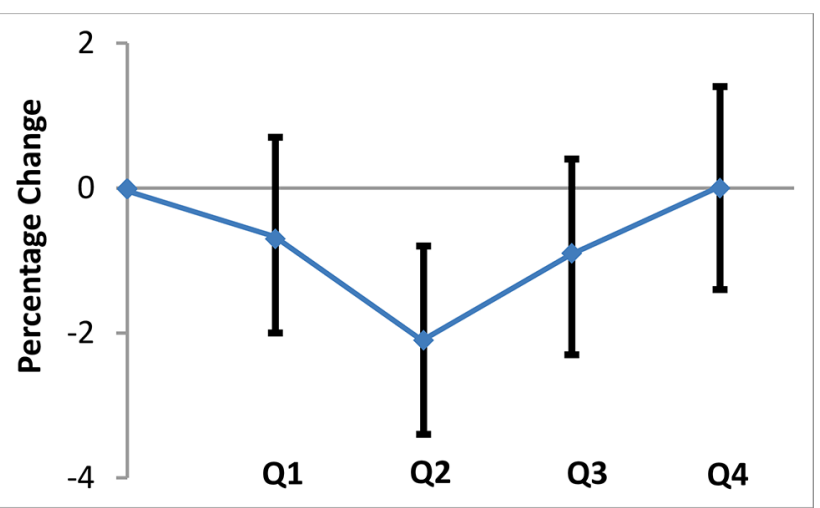

Figure 1 Percentage change and $95 \% \mathrm{Cl}$ in quarterly number of colonoscopies among physicians' patients following an adverse event experienced by a physician's patient.

The small decline in colonoscopy rates was evident for physicians' patients who were relatively younger (65-74 years) and older (75 years and older), suggesting that the decline was not related to specific consideration of an individual patient's risk of an adverse event (older patients experience less benefit from screening colonoscopy and have greater risks). Rather, physicians seem to have ordered fewer colonoscopies for all patients. Prior work suggests substantial overuse of colonoscopies in patients over the ages of 75 and 85 years. ${ }^{15} 16$ Nevertheless, fewer colonoscopies were performed overall among the older versus younger patients, which may reflect physicians' appreciation of the lower benefit of screening in this group. The decline in colonoscopy rates was observed for younger but not older physicians. Younger physicians, with less experience, may be particularly at risk of psychological biases associated with rare events.

We found no evidence of an effect on a physician's peers in a practice (those billing under the same tax identification number), suggesting that the impact of the negative adverse event was not sufficiently great as to influence practice-level discussions about screening. It

Table 3 Falsification test: change in quarterly number of mammograms among physicians' patients following an adverse colonoscopy event among a physician's patient*

\begin{tabular}{lcl}
\hline Quarter & \% change $(95 \% \mathbf{C l})$ & p Value \\
\hline 1 & $-0.4(-1.2$ to 0.3$)$ & 0.26 \\
2 & $-0.2(-0.9$ to 0.6$)$ & 0.66 \\
3 & $0.1(-0.6$ to 0.9$)$ & 0.74 \\
4 & $0.0(-0.7$ to 0.8$)$ & 0.95 \\
\hline
\end{tabular}

*Using fixed effects Poisson regression to model the number of mammograms. Models included fixed effects for each physician and indicators for study month as well as four indicator variables reflecting presence or absence of a colonoscopy adverse event in each of the four quarters before the month of interest. Models also include the number of patients assigned to the physician in that month, which serves as an offset variable allowing an interpretation of the dependent variable (number of mammograms) as a rate (number of mammograms per number of assigned patients). may be that physicians do not discuss such events or their thoughts about screening routinely with their practice partners. Alternatively, they may have such discussions with a limited group of colleagues, for which our method of identifying practice peers was not adequately sensitive.

Our findings suggest that efforts may be needed to help physicians avoid influences of psychological biases on the care they deliver. Decision-making is complex, and prior work suggests challenges in improving care delivery even after helping clinicians correct inaccurate estimation of the probability an event will occur. For example, one study succeeded in substantially improving clinicians' prior overestimations of the probability of streptococcal pharyngitis, but the proportion of patients prescribed antibiotics showed a trend towards increasing. ${ }^{17}$ Nevertheless, expanded use of shared decision-making tools holds great promise in helping physicians avoid cognitive biases in their estimates of probabilities of adverse events. If physicians and patients routinely discuss or review the benefits and harms of tests, procedures and treatments, then the associated probabilities and their expected implications will remain familiar to them. Decision aids can help with making such information easily accessible to patients and their physicians. ${ }^{18}$

Our study has several limitations. First, we focused on older Americans enrolled in fee-for-service Medicare; however, we do not expect the results to differ in other populations. We also studied only physicians caring for at least 25 Medicare beneficiaries, thus our findings may not generalise to very-low-volume physicians. Second, our evidence is indirect; we had no information about the physician's decision-making process (including the possible use of decision aids), if the assigned physician was the one who actually ordered the screening test, or the timing of colonoscopy orders for colonoscopies that were received. In addition, we inferred that these primary care physicians learnt about the serious adverse events, but we have no direct knowledge of this; nevertheless, such lack of awareness would tend to bias the results towards the null. We also did not observe colonoscopies that were ordered but not obtained by patients; nor did we observe changes to other screening strategies, such as faecal occult blood testing, which are not accurately identified in administrative data. ${ }^{19}$ In addition, we were not able to identify precisely patients who required more frequent colonoscopies per current screening guidelines. We therefore relied on the assumption that rates among a physician's panel would be relatively stable over time, consistent with prior studies. ${ }^{20} \mathrm{Next}$, there may have been some misattribution of patients to physicians, although we do not expect that would create any bias. Also, the relatively few serious adverse events observed, despite being consistent with prior studies, ${ }^{8}$ limited our power to assess for differences among physicians experiencing multiple adverse events. Finally, we did not attempt to distinguish between screening and diagnostic colonoscopies. While we might expect 
to see a greater decrease in screening colonoscopies following an adverse colonoscopy event because these may be less necessary, we also might also see a decline in diagnostic colonoscopies, which have higher baseline rates of adverse events. A new algorithm for identifying screening colonoscopies using claims data ${ }^{21}$ may allow for such distinctions once externally validated.

In conclusion, a physician's experience of a patient having a serious adverse event from colonoscopy was associated with a small and temporary decline in rates of colonoscopy among that physician's other patients that did not vary by the baseline risk of the physician's patients based on age, but was observed primarily for younger physicians, who have less clinical experience. These findings suggest that cognitive bias can lead some physicians to inaccurately interpret the relative harm to benefit ratio. Increased use of tools to enhance shared decision-making with patients may be one strategy to ensure that clinical decisions are based on the best available evidence about benefits and harms.

\section{Author affiliations}

${ }^{1}$ Department of Health Care Policy, Harvard Medical School, Boston, Massachusetts, USA

${ }^{2}$ Division of General Internal Medicine, Brigham and Women's Hospital, Boston, Massachusetts, USA

${ }^{3}$ The Department of Biomedical Data Science, The Dartmouth Institute for Health Policy and Clinical Practice, Geisel School of Medicine at Dartmouth, Hanover, Massachusetts, USA

${ }^{4}$ Department of Biostatistics, Harvard T.H Chan School of Public Health, Boston, Massachusetts, USA

${ }^{6}$ Division of General Medicine, Beth Israel Deaconess Medical Center, Boston, Massachusetts, USA

Acknowledgements The authors thank Laurie Meneades for expert statistical programming and Mary Hurley for administrative assistance. Ms Meneades and Ms Hurley are employed by Harvard Medical School and their work on this project was supported by research grants to Harvard Medical School from the National Cancer Institute. The corresponding author has the right to grant on behalf of all authors, and does grant on behalf of all authors, a worldwide licence (http://www.bmj.com/ sites/default/files/BMJ\%20Author\%20Licence\%20March\%202013.doc) to the publishers and its licensees in perpetuity, in all forms, formats and media (whether known now or created in the future), to (i) publish, reproduce, distribute, display and store the contribution; (ii) translate the contribution into other languages, create adaptations, reprints, include within collections and create summaries, extracts and/or abstracts of the contribution and convert or allow conversion into any format including without limitation audio; (iii) create any other derivative work(s) based in whole or part on the on the contribution; (iv) to exploit all subsidiary rights to exploit all subsidiary rights that currently exist or as may exist in the future in the contribution; (v) the inclusion of electronic links from the contribution to third-party material wherever it may be located; and (vi) license any third party to do any or all of the above. All research articles will be made available on an Open Access basis (with authors being asked to pay an open access fee-see http://www.bmj. com/about-bmj/resources-authors/forms-policies-and-checklists/copyright-openaccess-and-permission-reuse). The terms of such Open Access shall be governed by a Creative Commons licence- - details as to which Creative Commons licence will apply to the research article are set out in our worldwide licence referred to above.

Contributors NLK had full access to all of the data in the study and affirms that the manuscript is an honest, accurate and transparent account of the study being reported; that no important aspects of the study have been omitted and that any discrepancies from the study as planned (and, if relevant, registered) have been explained. Study concept and design, analysis and interpretation of data, critical revision of the manuscript for important intellectual content and final approval of the manuscript: all authors. Drafting of the manuscript: NLK.
Funding This work was supported by 1R01CA174468 from the National Cancer Institute. NLK is also supported by K24CA181510 from the National Cancer Institute.

Competing interests NLK previously served as a medical editor for the Informed Medical Decisions Foundation, now part of Healthwise, a non-profit organisation that seeks to improve healthcare decisions.

Ethics approval Harvard Medical School Committee on Human Studies.

Provenance and peer review Not commissioned; externally peer reviewed.

Data sharing statement Data were obtained from the US Centers for Medicare and Medicaid Services (CMS). Due to data use agreement restrictions, we cannot share our project data with other investigators, but the Medicare data can be obtained from CMS. Statistical code is available from the authors upon request.

Open Access This is an Open Access article distributed in accordance with the Creative Commons Attribution Non Commercial (CC BY-NC 4.0) license, which permits others to distribute, remix, adapt, build upon this work non-commercially, and license their derivative works on different terms, provided the original work is properly cited and the use is non-commercial. See: http://creativecommons.org/ licenses/by-nc/4.0/

(C) Article author(s) (or their employer(s) unless otherwise stated in the text of the article) 2017. All rights reserved. No commercial use is permitted unless otherwise expressly granted.

\section{REFERENCES}

1. Ramdass P, Petraro P, Via C, et al. Providers role in colonoscopy screening for colorectal Cancer. Am J Health Behav 2014; 38:234-44.

2. Laiyemo AO, Adebogun AO, Doubeni CA, et al. Influence of provider discussion and specific recommendation on colorectal Cancer screening uptake among U.S. adults. Prev Med 2014;67:1-5.

3. Zarychanski R, Chen Y, Bernstein CN, et al. Frequency of colorectal Cancer screening and the impact of family physicians on screening behaviour. CMAJ 2007;177:593-7.

4. Siegel RL, Miller KD, Jemal A, et al. Colorectal cancer statistics. CA Cancer J Clin 2016;66:7-30.

5. Welch HG, Robertson DJ. Colorectal Cancer on the Decline--why Screening Can't explain it all. N Engl J Med 2016;374:1605-7.

6. Bibbins-Domingo K, Grossman DC, Curry SJ, et al. Screening for colorectal Cancer: us preventive Services Task Force Recommendation Statement. JAMA 2016;315:2564-75.

7. Yabroff KR, Klabunde CN, Yuan G, et al. Are physicians' recommendations for colorectal Cancer screening guidelineconsistent? J Gen Intern Med 2011;26:177-84.

8. Warren JL, Klabunde CN, Mariotto AB, et al. Adverse events after outpatient colonoscopy in the Medicare population. Ann Intern Med 2009;150(12):849-57.

9. Tversky A, Kahneman D. Judgment under uncertainty: heuristics and biases. Science 1974;185:1124-31.

10. Tversky A, Kahneman D. Availability: a heuristic for judging frequency and probability. Cogn Psychol 1973;5:207-32.

11. Poses RM, Anthony M, Availability AM. Availability, wishful thinking, and physicians' diagnostic judgments for patients with suspected bacteremia. Med Decis Making 1991;11:159-68.

12. Mamede S, van Gog T, van den Berge K, et al. Effect of availability Bias and reflective reasoning on diagnostic accuracy among internal medicine residents. JAMA 2010;304:1198-203.

13. McWilliams JM, Hatfield LA, Chernew ME, et al. Early Performance of Accountable Care Organizations in Medicare. N Engl J Med 2016;374:2357-66.

14. Baldwin LM, Adamache W, Klabunde CN, et al. Linking physician characteristics and medicare claims data: issues in data availability, quality, and measurement. Med Care 2002;40:IV82-95.

15. Bian J, Bennett C, Cooper G, et al. Assessing colorectal Cancer screening adherence of Medicare Fee-for-Service beneficiaries Age 76 to 95 years. J Oncol Pract 2016;12:e670-e680.

16. Schwartz $A L$, Landon BE, Elshaug $A G$, et al. Measuring low-value care in Medicare. JAMA Intern Med 2014;174:1067-76.

17. Poses RM, Cebul RD, Wigton RS. You can lead a horse to water-improving physicians' knowledge of probabilities may not affect their decisions. Med Decis Making 1995;15:65-75.

18. Stacey D, Bennett CL, Barry MJ, et al. Decision aids for people facing health treatment or screening decisions. Cochrane Database Syst Rev 2011;10:CD001431.

19. Schenck AP, Klabunde CN, Warren JL, et al. Evaluation of claims, medical records, and self-report for measuring fecal occult blood 
testing among medicare enrollees in fee for service. Cancer Epidemiol Biomarkers Prev 2008;17:799-804.

20. Wharam JF, Zhang F, Landon BE, et al. Colorectal Cancer screening in a nationwide High-deductible Health Plan before and after the Affordable Care Act. Med Care 2016;54:466-73.
21. Adams KF, Johnson EA, Chubak J, et al. Development of an algorithm to classify colonoscopy indication from coded health care data. eGEMs (Generating evidence \& methods to improve patient outcomes). 2015;3. on http://repository.academyhealth.org/egems/ vol3/iss $1 / 11$ 